\title{
Transcriptional regulation of the aconitase genes (acnA and acnB) of Escherichia coli
}

\author{
Louise Cunningham, Megan J. Gruer and John R. Guest
}

Author for correspondence: John R. Guest. Tel: +44 114 2224406/3. Fax: +44 1142728697. e-mail: J.R.Guest@Sheffield.ac.uk

The Krebs Institute for Biomolecular Research, Department of Molecular Biology and

Biotechnology, University of Sheffield, Western Bank, Sheffield S10 2TN, UK

\begin{abstract}
Escherichia coli contains two differentially regulated aconitase genes, acnA and acnB. Two acnA promoters transcribing from start points located 407 bp $\left(P 1_{\text {acnA }}\right)$ and $50 \mathrm{bp}\left(P 2_{\text {acnA }}\right)$ upstream of the acnA coding region, and one acnB promoter $\left(P_{\text {acnB }}\right)$ with a start point 95 bp upstream of the acnB coding region, were identified by primer extension analysis. A $2.8 \mathrm{~kb}$ acnA monocistronic transcript was detected by Northern blot hybridization, but only in redoxstressed (methyl-viologen-treated) cultures, and a $2.5 \mathrm{~kb}$ acnB monocistronic transcript was detected in exponential- but not stationary-phase cultures. These findings are consistent with previous observations that $\operatorname{acn} A$ is specifically subject to SoxRS-mediated activation, whereas acn $B$ encodes the major aconitase that is synthesized earlier in the growth cycle than AcnA. Further studies with acn-lacz gene fusions and a wider range of transcription regulators indicated that acnA expression is initiated by $\sigma^{38}$ from $P_{1}{ }_{\text {acnA }}$ and from $P 2_{\text {acnA }}$ it is activated directly or indirectly by CRP, FruR, Fur and SoxRS, and repressed by ArCA and FNR. In contrast, acnB expression is activated by CRP and repressed by ArcA, FruR and Fis from $P_{\text {acnB }}$. Comparable studies with fum-lacZ fusions indicated that transcription of fumC, but not of fumA or fumB, is initiated by RNA polymerase containing $\sigma^{38}$. It is concluded that AcnB is the major citric acid cycle enzyme, whereas AcnA is an aerobic stationaryphase enzyme that is specifically induced by iron and redox-stress.
\end{abstract}

Keywords: aconitase, fumarase, citric acid cycle genes, transcription regulation, global regulators

\section{INTRODUCTION}

Aconitase (EC 4.2.1.3) catalyses the reversible isomerization of citrate and isocitrate via cis-aconitate in the citric acid cycle. It is a monomeric enzyme containing an unstable $[4 \mathrm{Fe}-4 \mathrm{~S}]$ cluster which is reversibly converted to an inactive [3Fe-4S] form (Beinert et al., 1996). The aconitase protein family contains aconitases, isopropylmalate isomerases and homoaconitases from diverse sources, and the iron-dependent regulatory proteins (IRP) which regulate either the translation or the stabilities of specific vertebrate mRNAs (Klausner \& Rouault, 1993; Frishman \& Hentze, 1996; Hentze \& Kuhn, 1996; Gruer et al., 1997a). The bifunctional IRP1 proteins, which operate either as cytoplasmic aconitases or as site-specific RNA-binding proteins depending on the reversible incorporation or disruption of the iron-sulphur clusters, are particularly interesting. A structural archetype for all members of this family is provided by the porcine mitochondrial aconitase (Robbins \& Stout, 1989). The basic structure contains three structural domains, tightly packed around the iron-sulphur cluster, that are located beneath a deep active-site cleft and the upper (fourth) domain. However, three different domain arrangements have now been recognized (Gruer et al., 1997a). In most cases domain four is at the C-terminal end, connected to the rest of the molecule by a long linker, but in some bacterial aconitases (notably AcnB of Escherichia coli, see below) domain four is located at the N-terminus, and in the heterodimeric bacterial isopropylmalate isomerases the fourth domain is provided by an independent subunit.

Biochemical and genetic studies have established that $E$. coli contains two genetically distinct aconitases (designated AcnA and AcnB) encoded by the $a c n A$ and $a c n B$ genes (Prodromou et al., 1991; Bradbury et al., 1996). There may even be a third aconitase $(\mathrm{AcnC})$ responsible for the low residual aconitase activity detected in a genetically engineered $a c n A$ acnB double mutant (Gruer et al., 1997b). The acnA gene is located at $28 \mathrm{~min}$ 
Table 1. E. coli strains and plasmids

\begin{tabular}{|c|c|c|}
\hline Strain or plasmid & Relevant characteristic & Source or reference ${ }^{*}$ \\
\hline W3110 & Prototroph & Laboratory collection \\
\hline DH5 $\alpha$ & $\Delta(\operatorname{argF-lac}) U 169(\phi 80 \Delta l a c Z M 15)$ recA & Sambrook et al. (1989) \\
\hline MC1000 & $\Delta l a c X 74 \Delta($ araA-leu $)$ & Silhavy et al. (1984) \\
\hline JRG1999 & $\mathrm{MC1000 \Delta crpT8}$ & S. T. Cole ${ }^{1}$ \\
\hline RK4353 & $\Delta(\operatorname{argF}-\operatorname{lac}) \cup 169$ & V. Stewart ${ }^{2}$ \\
\hline RK5279 & RK4353 fnr-250 & V. Stewart ${ }^{2}$ \\
\hline RJ1802 & RK4353 fis-767 & Ball et al. (1992) \\
\hline JRG3227 & RK4353 fruR & Park et al. (1995) \\
\hline MC4100 & $\Delta(\operatorname{argF}-\mathrm{lac}) U 169 r p s L$ & Silhavy et al. (1984) \\
\hline H1941 & MC4100 $\Delta$ fur & K. Hantke ${ }^{3}$ \\
\hline LM1A & MC4100 arcA1 zjj::Tn10 & McWalter et al..$^{4}$ \\
\hline RM315 & MC4100 $\Delta f n r$ arcA1 zjj:: $\operatorname{Tn} 10$ & $\begin{array}{l}\text { Sawers \& Suppmann } \\
\text { (1992) }\end{array}$ \\
\hline RH90 & MC4100 rpoS359:: Tn10 & R. Hennge-Aronis ${ }^{5}$ \\
\hline GC4468 & $\Delta(\operatorname{argF}-\mathrm{lac}) U 169 \mathrm{rpsL}$ sup & Greenberg et al. (1990) \\
\hline DJ901 & GC4468 $\Delta$ soxRS & Greenberg et al. (1990) \\
\hline pGS447 & pUC119, acn $A^{+}, \mathrm{Ap}^{\mathrm{R}}$ & Prodromou et al. (1991) \\
\hline pGS782 & pUC119, $a c n B^{+}, \mathrm{Ap}^{\mathrm{R}}$ & Bradbury et al. (1996) \\
\hline pGS279 & $c r p^{+}, \mathrm{Ap}^{\mathrm{R}} \mathrm{Cm}^{\mathrm{R}}$ & Spiro et al. (1989) \\
\hline $\mathrm{pCH} 21$ & $f n r^{+}, \mathrm{Ap}^{\mathrm{R}} \mathrm{Cm}^{\mathrm{R}}$ & C. H. Higgins ${ }^{6}$ \\
\hline pRB38 & $\operatorname{arc} A^{+}, \mathrm{Cm}^{\mathrm{R}}$ & R. Buxton? \\
\hline pMH15 & $f u r^{+}, \mathrm{Cm}^{\mathrm{R}}$ & K. Hantke ${ }^{3}$ \\
\hline pGS824 & $r p o S^{+}, \mathrm{Cm}^{\mathrm{R}}$ & This work \\
\hline$\lambda \mathrm{G} 185$ & fumA-lacZ fusion & Woods \& Guest (1987) \\
\hline$\lambda \mathrm{G} 186$ & fumB-lacZ fusion & Woods \& Guest (1987) \\
\hline$\lambda \mathrm{G} 187$ & fum $\mathrm{C}-$ lacZ fusion & Woods \& Guest (1987) \\
\hline$\lambda \mathrm{G} 244$ & $\operatorname{acn} A-\operatorname{lac} Z$ fusion & Gruer \& Guest (1994) \\
\hline$\lambda \mathrm{G} 259$ & $a c n B-l a c Z$ fusion & Gruer et al. (1997b) \\
\hline
\end{tabular}

* 1, Pasteur Institute, Paris, France; 2, Cornell University, Ithaca, NY, USA ; 3, University of Tubingen, Germany; 4, University of Dundee, UK; 5, University of Konstanz, Germany; 6, Imperial Cancer Research Fund, John Radcliffe Hospital, Oxford, UK; 7, National Institute for Medical Research, Mill Hill, London, UK.

$(1350 \mathrm{~kb})$ in the E. coli chromosome (see Fig. 1), whereas the $a c n B$ gene is situated at $2.85 \mathrm{~min}(131 \mathrm{~kb})$. The AcnA protein exhibits a remarkable $53 \%$ sequence identity to the bifunctional IRP1 proteins, compared to $29 \%$ identity to the mitochondrial aconitases, and only $17 \%$ to $A c n B$, the most remote member of the aconitase family. Growth tests have suggested that an acnA mutation is complemented by the resident $a c n B^{+}$gene (because it fails to generate a distinct phenotype) whereas an $a c n B$ mutation produces a mutant phenotype which is only partially complemented by the $a c n A^{+}$gene (Gruer et al., 1997b). Enzymological and regulatory studies with acn-lacZ fusions have further indicated that the synthesis of both enzymes is subject to catabolite and anaerobic repression, and that $A \mathrm{cnB}$ is the major citric acid cycle enzyme, whereas AcnA is more important in stationary phase (Gruer \& Guest, 1994; Gruer et al., 1997b). Thus it was observed that the aconitase activity of an $a c n B$ mutant is reduced to onethird of the wild-type level whereas that of an $a c n A$ mutant is little affected. It was also observed that an $a c n B-l a c Z$ fusion is strongly expressed in the expo- nential phase whereas a comparable acnA-lac Z fusion is not expressed until early stationary phase (and then only weakly). Furthermore, the $a c n A-l a c Z$ fusion was specifically induced by redox-stress and by iron via SoxRSand Fur-activated regulatory mechanisms. Despite its close similarity to IRP1, there is as yet no evidence to suggest that AcnA performs a redox- or iron-dependent regulatory function.

To better understand the relative roles of $A c n A$ and $A c n B$, the transcriptional organization of the corresponding acn genes was investigated by Northern blot analysis and primer extension mapping. The responses of an acnB-lacZ translational fusion to a variety of transcriptional regulators were also investigated and compared to those of an analogous acnA-lacZ fusion. This showed that the $a c n B$ gene is subject to repression by ArcA, Fis and FruR and activation by CRP, whereas the acnA gene is repressed by ArcA and FNR, activated by FruR, CRP, Fur and SoxRS, and initiated by $\sigma^{38}$ in stationary phase. 


\section{METHODS}

Bacterial strains, plasmids and bacteriophages. The strains of E. coli $\mathrm{K}-12$ are listed in Table 1 . The source of acnA DNA was pGS447 (Prodromou et al., 1991) and the corresponding $\lambda$ acnA-lacZ translational fusion phage, $\lambda$ G244 (Fig. 1a), was constructed previously (Gruer \& Guest, 1994). The plasmid containing the entire $a c n B$ coding region (pGS782; Fig. 1b) was constructed previously by ligating a PCR-amplified fragment into pUC119 (Bradbury et al., 1996) and the $\lambda a c n B-$ lac $Z$ translational fusion phage ( $2 \mathrm{G} 259$; Fig. 1b) was also constructed previously (Gruer et al., 1997b). The rpoS multicopy plasmid (pGS824) was constructed by ligating a $4.3 \mathrm{~kb}$ EcoRI-HindIII fragment containing the $r p o S$ gene from pMMkatF2 (Mulvey et al., 1988) into pSU18 (Bartolomé et al., 1991). Other plasmids and phages are listed in Table 1.

The $\lambda a c n-l a c Z$ fusion phages were established as single-copy prophages in different sets of host strains for investigating the roles of global regulators in acn gene expression. Lysogens were selected initially with $\lambda b 80 \mathrm{de} l 9 c$ and monolysogens were identified by their sensitivity to $\lambda c 190 c 17$ and $\lambda v i r$. As described in previous studies with the $\lambda a c n A-l a c Z$ fusion phage (Gruer \& Guest, 1994), sets of $\lambda a c n B-$ lacZ monolysogens were assembled for each regulator, consisting of a parental strain, a regulator mutant, and a derivative of the latter transformed with a multicopy plasmid expressing the wild-type regulator gene, thus: CRP [MC1000, JRG1999, and JRG1999(pGS279)]; ArcA [MC4100, LM1A, and LM1A(pRB38)]; FNR [RK4353, RK5279, and RK5279(pCH21)]; FNR + ArcA [MC4100, RM315(fnr arcA), and RM315(pRB38) or RM315(pCH21)]; Fur [MC4100, H1941, and H1941(pMH15)] and $\sigma^{38}$ [MC4100,
RH90, and RH90(pGS824)]. For Fis and FruR, $\lambda a c n B-l a c Z$ monolysogens of the parental strain (RK4353) and respective regulator mutants (RJ1802 and JRG3227) were used. Analogous sets of monolysogens were isolated for the FNR + ArcA, $\sigma^{38}$, Fis and FruR series with $\lambda a c n A-$ lacZ ( $\left.\lambda \mathrm{G} 244\right)$, and for the $\sigma^{38}$ series with three $\lambda$ fum-lac $Z$ fusion phages: $\lambda$ fum $A-l a c Z$ ( $\lambda \mathrm{G} 185)$; $\lambda$ fumB-lacZ ( $\lambda \mathrm{G} 186)$ and $\lambda$ fumC-lacZ ( $\lambda \mathrm{G} 187)$, as previously described (Gruer \& Guest, 1994).

Microbiology and enzymology. The basic microbiological methods and media have been described previously (Gruer et al., 1997b). $\beta$-Galactosidase was assayed according to Miller (1972) using monolysogens containing lac $Z$ translational fusion phages grown to late exponential phase for $\lambda a c n B-l a c Z$ and $\lambda$ fum-lacZ, or early stationary phase for $\lambda a c n A-l a c Z$, in unsupplemented $\mathrm{L}$ broth or $\mathrm{L}$ broth containing glucose $(1 \%)$. In experiments involving SoxRS regulation, redox-stress was imposed by adding methyl viologen $\left(0.2 \mathrm{mg} \mathrm{ml}^{-1}\right.$, final concn) $1 \mathrm{~h}$ before the cultures were sampled, and in Fur studies, available iron was depleted by adding bipyridyl $(200 \mu \mathrm{M}$, final concn) at $\mathrm{OD}_{650} 0 \cdot 6,1 \mathrm{~h}$ before sampling. Activities (Miller units) were averaged from at least four independent cultures, the variation between samples being $<10 \%$.

DNA manipulation, RNA extraction, Northern hybridization and primer extension analysis. E. coli DH5 $\alpha$ was the routine transformation host (Table 1) and DNA was prepared and manipulated by standard procedures (Sambrook et al., 1989). RNA was extracted by the hot acid phenol method (Aiba et al., 1981) from bacteria grown in $\mathrm{L}$ broth to exponential phase $\left(\mathrm{OD}_{650} 0 \cdot 65\right)$ and early stationary phase $\left(\mathrm{OD}_{650} 2 \cdot 0\right)$ : where stated, methyl viologen $\left(0 \cdot 2 \mathrm{mg} \mathrm{ml}^{-1}\right)$ was added $1 \mathrm{~h}$ before

(a)

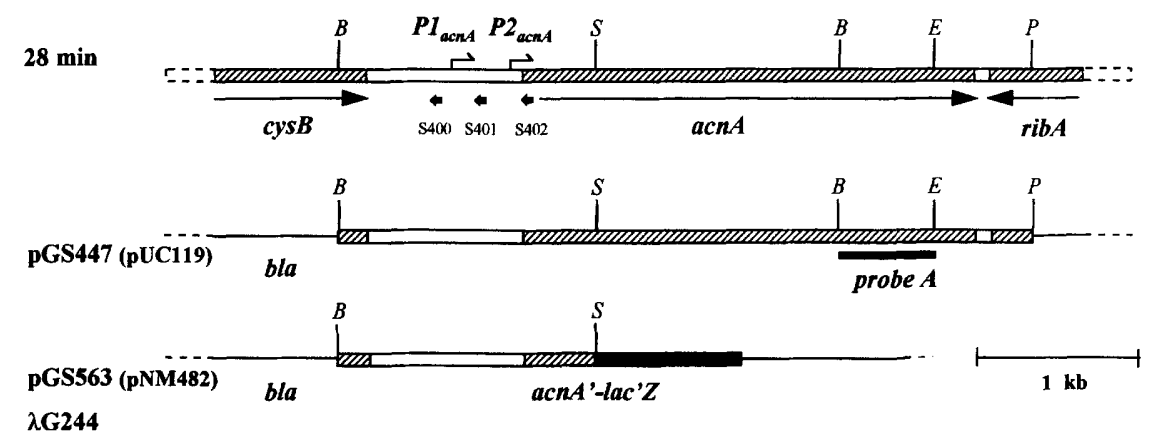

(b)

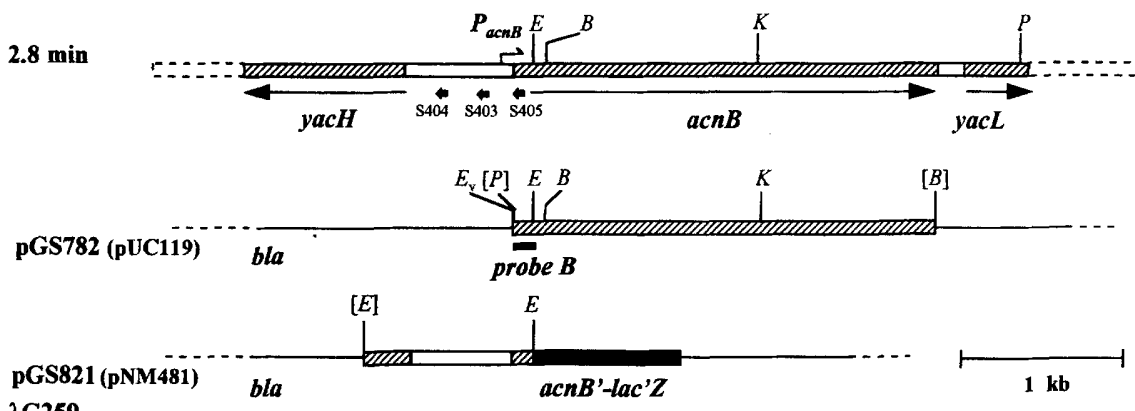

$\lambda \mathbf{G 5 9}$

Fig. 1. Restriction maps of the $a c n A$ and $a c n B$ regions showing the segments of DNA cloned in specific plasmids, the probes used for Northern blot analysis, the oligonucleotides used in primer extension analysis and the structures of the acnA-lacZ and acnB-lacZ fusion phages. Relevant restriction sites are indicated: B, BamHI; E, EcoRI; K, Kpnl; P, Pstl; S, Sall. The subscript $\left({ }_{v}\right)$ refers to a flanking vector site and engineered sites (Bradbury et al., 1996) are enclosed in square brackets. 
sampling. Bacteria equivalent to $50 \mathrm{ml}$ exponential culture $\left(\mathrm{OD}_{650} 0.65\right)$ were resuspended in $3 \mathrm{ml} 20 \mathrm{mM}$ sodium acetate (pH 5.5) containing $0.5 \%$ SDS and $1 \mathrm{mM}$ EDTA, and extracted at $60^{\circ} \mathrm{C}$ with phenol (equilibrated with $20 \mathrm{mM}$ sodium acetate, $\mathrm{pH} 5 \cdot 5)$. The phenol extraction of the aqueous extract was repeated, and the RNA recovered after three successive ethanol precipitations was dissolved in diethylpyrocarbonatetreated water.

Samples of RNA $(20 \mu \mathrm{g})$ denatured with formamide and formaldehyde, were fractionated electrophoretically in $1 \%$ agarose-formaldehyde gels with MOPS buffer and transferred to nylon membranes using $10 \times$ SSC (Sambrook et al., 1989). Hybridization with $\left[\alpha-{ }^{32} \mathrm{P}\right] \mathrm{dCTP}-$ labelled probes was as instructed in the Pharmacia 'Ready to Go' labelling kit. The probe for acnA transcripts was a $0.4 \mathrm{~kb}$ EcoRI-BamHI fragment of pGS447 (probe $A$ in Fig. 1a) and the $a c n B$ probe was a $0.1 \mathrm{~kb}$ EcoRI fragment of pGS782 (probe $B$ in Fig. 1b).

Primer extension analysis was performed according to Gerischer \& Dürre (1992) but modified to allow continuous incorporation of $\left[\alpha_{-}^{32} \mathrm{P}\right] \mathrm{dCTP}$. Total RNA $(100 \mu \mathrm{g})$ was annealed with $10 \mathrm{pmol}$ primer in $7 \mu \mathrm{l} 10 \mathrm{mM}$ Tris $/ \mathrm{HCl}$ (pH 8.3) plus $5 \mathrm{mM} \mathrm{KCl}\left(80^{\circ} \mathrm{C}\right.$ for $5 \mathrm{~min}$ and cooled slowly), before incubating for $10 \mathrm{~min}$ at $37^{\circ} \mathrm{C}$ with $1.5 \mu$ l reverse transcriptase buffer (Life Sciences), $0.5 \mu \mathrm{l}$ actinomycin D $\left(500 \mu \mathrm{g} \mathrm{ml} \mathrm{m}^{-1}\right), \quad 0.5 \mu \mathrm{l}$ placental ribonuclease inhibitor (Promega), $0.5 \mu$ l sodium pyrophosphate $(80 \mathrm{mM}), 3 \mu \mathrm{l}$ of a labelling mix containing dATP, dGTP and TTP (each at $5 \mu \mathrm{M})$ plus $\left[\alpha^{-32} \mathrm{P}\right] \mathrm{dCTP}(9 \mu \mathrm{M})$, and $2 \mu \mathrm{l}$ AMV reverse transcriptase (50 U; Life Sciences). Thereafter, $3 \mu \mathrm{l}$ of a solution containing the four dNTPs (each at $5 \mathrm{mM}$ ) was added and incubation continued for a further $90 \mathrm{~min}$ at $37^{\circ} \mathrm{C}$. The reactions were stopped with $1 \mu$ IDTA $(0.5 \mathrm{M})$, treated with RNase A $\left(50 \mu \mathrm{g} \mathrm{ml}^{-1}\right)$ for $5 \mathrm{~min}$, and $150 \mu \mathrm{l}$ TE was added prior to phenol/chloroform extraction and ethanol precipitation. Samples in $4 \mu \mathrm{l}$ TE plus $3 \mu \mathrm{l}$ Sequenase Stop Solution were fractionated by electrophoresis in $6 \%$ acrylamide $/ 7 \mathrm{M}$ urea gels alongside a sequence ladder derived from the corresponding DNA and primer. The oligonucleotide primers (see Fig. 1) were: S400 (588-560); S401 (990-961) and S402 (1319-1288) for acnA (numbered according to Prodromou et al., 1992) and S405 (20324-20295); S404 (19576-19551) and S403 (19950-19925) for acnB (numbered according to Fujita et al., 1994).

Materials. Restriction enzymes were from Northumbria Biologicals. AMV reverse transcriptase was from Life Sciences. Radiolabelled $\left[\alpha^{32} \mathrm{P}\right] \mathrm{dCTP}\left(110 \mathrm{TBq} \mathrm{mmol}^{-1}\right)$ and nylon membranes were from Amersham and the RNA calibration ladder was from Life Technologies.

\section{RESULTS}

\section{Transcript analysis for the acnA gene}

Northern blot hybridization was used to detect acnA transcripts in RNA from W3110 (wild-type) using a $400 \mathrm{bp}$ segment of the acnA coding region as the probe (Fig. 1a). A transcript of approximately $2 \cdot 8 \mathrm{~kb}$ was detected in RNA from cultures that had been redoxstressed by treating with methyl viologen $1 \mathrm{~h}$ before reaching stationary phase, but not in untreated stationary-phase cultures (Fig. 2a). A transcript of the same size was just detectable in cultures that had been similarly stressed and sampled whilst still in exponential phase, but again no transcript could be detected in comparable untreated cultures (data not shown), nor in stationary-phase cultures grown with extra iron, $83 \mu \mathrm{M}$ (data not shown). These observations are consistent with those made previously with an acnA-lacZ fusion which indicated that $a c n A$ gene expression is low, even at its highest level in stationary phase, but can be increased in a soxRS-dependent manner by methylviologen-induced redox-stress (Gruer \& Guest, 1994).

Transcriptional start sites for the acnA gene were defined by primer extension analysis using RNA from early-stationary-phase cultures of W3110 and three primers spanning the $1003 \mathrm{bp} c y s B-a c n A$ intergenic region (Fig. 1a). Two promoters, $P 1_{\text {acna }}$ and $P 2_{\text {acnA }}$, were identified by a pair of start sites 407 and $403 \mathrm{bp}$ upstream of the $a c n A$ coding region and a single start site $50 \mathrm{bp}$ upstream of the coding region, respectively (Fig. 3a, b; Fig. 4a). The same set of start sites was observed with RNA samples from both redox-stressed and unstressed stationary-phase cultures, despite the failure to detect transcripts in the latter samples by hybridization (Table 2). It is presumed that transcripts of low abundance are more readily detected by primer extension analysis. In contrast, primer extension analysis with RNA from exponential-phase cultures indicated that $P 2_{\text {acnA }}$ is active in both redox-stressed and unstressed cultures but no evidence was found for transcription from $P 1_{\text {aena }}$ in either type of exponential-phase culture (Table 2). This suggests that the higher level of acnA-lac Z expression observed in stationary phase may be generated from $P 1_{\mathrm{acnA}}$. No other transcriptional start sites having the same clockwise polarity were detected in the long intergenic region upstream of $P 1_{\mathrm{acnA}}$. When the sizes predicted for the corresponding monocistronic acnA transcripts, 3.130 and $3.126 \mathrm{~kb}$ from $P 1_{\mathrm{acnA}}$ and $2.773 \mathrm{~kb}$ from $P 2_{\mathrm{acnA}}$, are compared with the relative activities of the promoters under different conditions (Table 2), it is concluded that $P 2_{\text {acn }}$ is the source of the major transcript $(2.8 \mathrm{~kb}$ approx.) detected in redoxstressed cultures.

The slightly shorter and least abundant transcript generated by $P 1_{\text {acna }}$ probably arises by primer slippage during RNA chain initiation. This is because initiation normally involves the formation of many short, loosely bound, abortive transcripts ( $\leqslant 9$ nucleotides), which can be translocated before a productive transcript escapes the promoter (Chamberlin \& Hsu, 1996). As can be seen in Fig. 4(a), a 5-nucleotide transcript initiating at the upstream site (407 bp from the coding region) and having TG(UG) at its $3^{\prime}$ end, might translocate $4 \mathrm{bp}$ downstream to a position where the TG motif is repeated. This would generate a transcript corresponding to the minor transcript which appears to have initiated 4 bp downstream from the major site. The corresponding promoter region lacks -35 and -10 hexamers resembling those of $\sigma^{70}$ - or $\sigma^{38}$-dependent promoters. Instead, it contains sequences that more closely resemble a gearbox promoter (Vicente et al., 1991), CTGgcA and CaGCAAGcc separated by $13 \mathrm{bp}$ (Fig. 4a), compared to the gearbox consensus : CTGCAA and CGGC ${ }_{T}$ AGTA separated by 14 to $16 \mathrm{bp}$. 
(a)

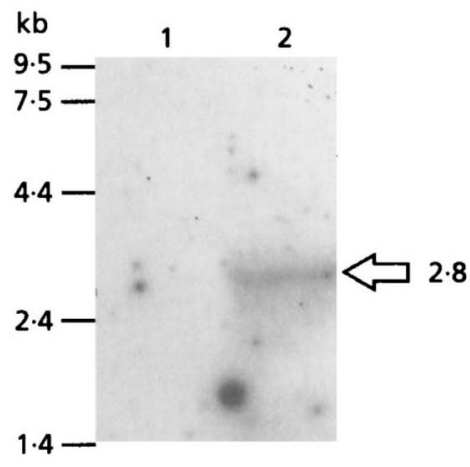

(b)

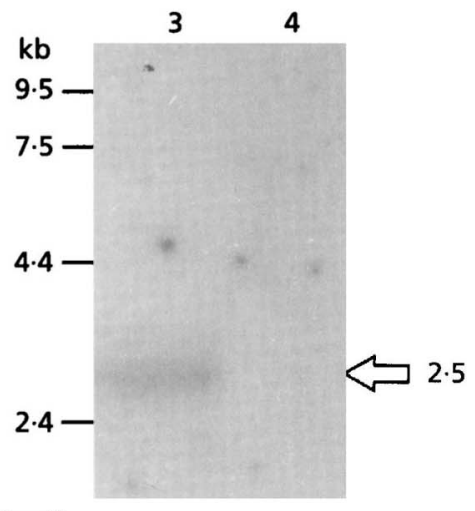

Fig. 2. Northern blot hybridization showing transcripts of the aconitase genes. (a) The acnA probe was used with RNA extracted from cultures of W3110 entering stationary phase in the absence or presence of methyl viologen (lanes 1 and 2, respectively). (b) The acn $B$ probe was used with RNA extracted from exponential-phase or earlystationary-phase cultures of W3110 (lanes 3 and 4, respectively). The RNA standards (kb) provide calibrations for the hybridizing transcripts (arrowed). (a)

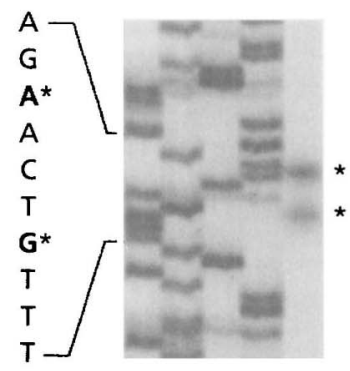

(b)

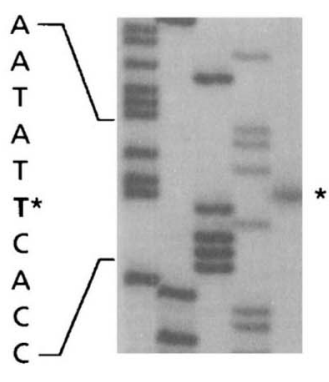

(c)

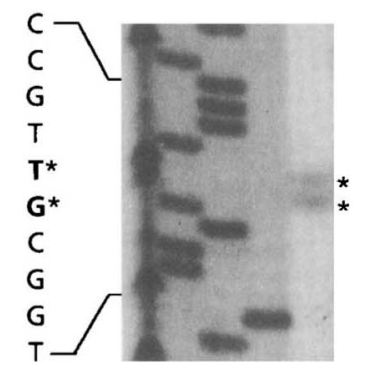

Fig. 3. Primer extension mapping of the transcriptional start sites of the acn genes. The start sites (*) associated with specific promoters are shown with sequence ladders for their location in Fig. 4: (a) $P 1_{\text {acnA }}$, (b) $P 2_{\text {acna, }}$ and (c) $P_{\text {acns. }}$. In (a) there is a $0.5 \mathrm{bp}$ discrepancy between the sequence ladder and the primer extension products, but the validity of the interpretation is supported by other experiments.
The second acnA promoter region $\left(P 2_{\text {acnA }}\right)$ contains a well-placed -10 hexamer which matches the $\sigma^{70}$ consensus at four positions, but there is no corresponding -35 hexamer (Fig. 4a). However, it resembles an 'extended -10 promoter' because the -10 hexamer is preceded by a TGn motif, which is known to increase promoter strength and even to allow transcription initiation in the absence of a -35 hexamer (Kumar et al., 1993). Another potential $P 2_{\text {aenA }}$ promoter sequence contains -35 (TTATCA) and -10 (TGTTAT) hexamers (marked by dashed overlines in Fig. 4a), but these are sub-optimally separated from each other $(19 \mathrm{bp})$ and from the transcription start site $(9 \mathrm{bp})$.

\section{Transcript analysis for the acnB gene}

Northern blot hybridization was used with a probe from the $5^{\prime}$ end of the $a c n B$ coding region (Fig. 1b) for analysing $a c n B$ transcription. A single transcript of approximately $2.5 \mathrm{~kb}$ was detected in samples of RNA from exponential-phase cultures but no transcripts could be found in early-stationary-phase samples (Fig. $2 \mathrm{~b})$. This pattern of expression was unaffected by redoxstress (data not shown), and it is consistent with previous observations that $A \mathrm{cnB}$ activity and $a c n B$ expression increase early in the growth cycle but decline in stationary phase (Gruer et al., 1997b).

Primer extension analysis with RNA from exponentialphase cultures and three different primers designed to span the $y a c H-a c n B$ intergenic region (Fig. $1 b$ ) indicated that acnB transcription is initiated 96 and 95 bp up- stream of the coding region (Figs $3 c, 4 b$ ). The sizes predicted for the monocistronic acnB transcripts extending to a putative rho-independent $a c n B$ terminator are 2.761 and $2.760 \mathrm{~kb}$, in reasonable agreement with the $2.5 \mathrm{~kb}$ transcript detected by Northern blotting. The start sites are preceded by a potential promoter, $P_{\mathrm{acnB}}$, having -35 and -10 hexamers differing at only one and two positions (respectively) from those of the canonical $\sigma^{70}$ sequences (Fig. $4 b$ ). The -10 region also resembles that of an 'extended -10 promoter' in which the -10 hexamer is preceded by a TGn motif (Kumar et al., 1993).

\section{Studies with acn-lacz translational fusions}

Studies on the expression of individual acn genes were made with single-copy $a c n-l a c Z$ translational fusions in which the acnA or $a c n B$ promoters accompanied by parts of the corresponding coding regions are linked 'in phase' to the lac $Z$ coding region and transferred to phage $\lambda: \lambda G 244, \lambda a c n A-l a c Z$ and $\lambda G 259, \lambda a c n B-l a c Z$ (Table 1, Fig. 1). The fusion phages were established as prophages in a variety of lac $Z$ deletion hosts such that the $\beta$-galactosidase activities reflect the overall changes in the transcription and translation of the acn genes. The effects of global regulators were generally assessed by comparing a parental monolysogen, $a c n A^{+} B^{+} \Delta l a c$ ( $2 a c n-l a c Z$ ), with derivatives lacking the regulator, and others containing multiple copies of the regulatory gene.

Regulation of acn gene expression by CRP. Previous studies indicated that $A c n A$ and $A c n B$ are both subject to 
(a) $\operatorname{acn} A$

GTCAGGAGCA ACTGGAGTCG TCACCATTAT GCCAGCATAG TGACAATGAA $-60 \quad-40 \quad-35$ gearbox

ACAGAAACGA AACGGGAATG TTCCGTCGTT ATTCCAGACG AqTGGCAACT

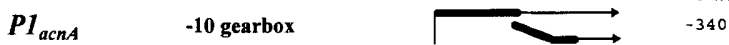
AACATCGCAG CAGCAAGCOT TTATAGAACT GTTTGCTGAA GATGATCAGC $-320 \quad-300$

CGAAACAATA ATTATCATCA TTCTTATTAC CCATTTTTAA TGAATTAAAG $-270 \quad-250$ FNR

GGCTTITAAT ACACCGCAGC AATAACAGCT TGAGTTATCT CAACACAAAA $-220 \quad-200$

TAATAACCGT TAAGGGTGTA GCCTATGATC AACACAAATA TGAAATATTG $-170 \quad-150$

GTCCTGGATG GGCGCGTTTT CTCTGTCGAT GCTCTTCTGG GCCGAACTCC $-220$ $-100$

TCTGGATCAT TACTCACTGA TCCTTGACCC CGCTGCGGCG GGGTTGTCAT

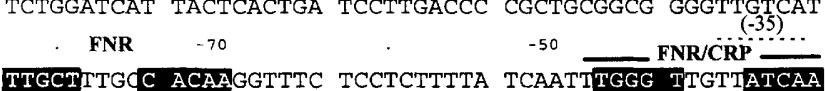
$\begin{array}{llll}P 2_{a c n A} & -20 & (-10) & -10\end{array} \longrightarrow$ ArCA ĀTCGTTACGC GATGTTTGTG TTATCTTTAA TATTCACCCT GAAGAGAATC

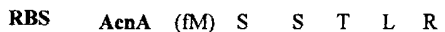
AGGGCTTCGC AACCCTGTCA TAAGGAGGA GCTATGTCGT CAACCCTACG

(b) $a c n B$

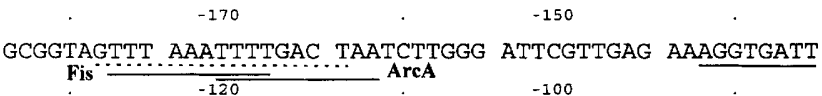
ATCACCATGC GAATTAACGA AGTTTTTACG GAGGGAAACA ATCTCTAGAC - $\frac{1}{-70}$ ArcA $\quad-50 \quad$ CRP CATCCTTAAC GATTCAGCCA CTTTTTTATG TTGCTTTTTT GTAAACAGA $\begin{array}{rlll}-35 & \text { Fis } & -20 P_{\text {acnB }} & -10 \longrightarrow\end{array}$

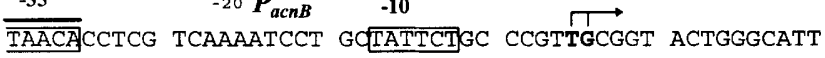
Fis

TACCCTACAA ACTGCTGTCT CACAGGAGCG TGAAGAGAAT CGCCTGCCGC RBS $\quad \begin{array}{lllllllll}\text { AcnB } & \text { (fM) } & \text { L } & \text { E } & \text { E } & Y & R & K\end{array}$ ACTATGACAA TGAGAGCGAG GAGAACCGTC GTGCTAGAAG AATACCGTAA

Fig. 4. Nucleotide sequences upstream of the acnA- and $a c n B$ coding regions. The transcriptional start sites and translational initiation sites (bold), the -35 and -10 hexamers of putative promoters (boxed), alternative hexamers for $P 2_{\text {acnA }}$ (dashed overlined), ribosome binding sites (RBS), and potential binding sites for FNR (black box), CRP (bold overlined), ArcA (underlined) and Fis (dashed underlined), are shown for (a) acnA (co-ordinates 701-1250; Prodromou et al., 1992) and (b) acnB (co-ordinates 19951-20250; Fujita et al., 1994).

catabolite repression, and evidence for the CRP-dependent activation of the acnA gene came from the 3.5fold lowering of acnA-lac $Z$ expression in a crp mutant and the enhanced activity after transformation with a $c r p^{+}$multicopy plasmid (Gruer \& Guest, 1994; Gruer et al., 1997b). The activity of the single-copy acnB-lacZ fusion was likewise shown to be reduced $2 \cdot 7$-fold in a $c r p$ deletion strain and restored (but not enhanced) by the multicopy $\mathrm{crp}^{+}$plasmid (Fig. 5a). The activities of the crp $p^{+}$strains were also reduced approximately twofold by adding glucose $(1 \%)$. Thus it was concluded that the cAMP-CRP complex is required to activate transcription of both the $a c n A$ and $a c n B$ genes.
Table 2. Primer extension analysis of transcription from two acnA promoters

The results of primer extension analysis are summarized for $P 1_{\text {acnA }}$ and $P 2_{\text {acnA }}$ using RNA extracted from L-broth cultures harvested in exponential phase $\left(\mathrm{OD}_{650} 0.65\right)$ or early stationary phase $\left(\mathrm{OD}_{650} 2 \cdot 0\right)$ without $(-)$ and with $(+\mathrm{MV})$ the addition of methyl viologen $\left\{0.2 \mathrm{mg} \mathrm{ml}^{-1}\right) 1 \mathrm{~h}$ before harvesting. The relative amounts of product formed $(-1+1+++)$ refer to individual promoters, not to comparisons between promoters.

\begin{tabular}{|lccccc|}
\hline Promoter & \multicolumn{2}{c|}{ Exponential phase } & \multicolumn{2}{l|}{ Stationary phase } \\
\cline { 2 - 3 } \cline { 5 - 6 } & - & $+\mathrm{MV}$ & & - & $+\mathrm{MV}$ \\
\hline$P 1_{\mathrm{acnA}}$ & - & - & & + & + \\
$P 2_{\mathrm{acnA}}$ & + & + & & + & +++ \\
\hline
\end{tabular}

Regulation of acn gene expression by SoxRS and Fur. The acnA gene was previously shown to be induced by redox-stress via the SoxRS system, repressed by irondepletion, and activated by the ferric uptake regulator, Fur (Gruer \& Guest, 1994). In contrast, expression of the $a c n B$ gene appeared to be essentially independent of these regulatory mechanisms. The activity of the acnB-lacZ fusion was little affected by soxRS deletion (Fig. 5b); the inducing effects of methyl viologen, particularly apparent in the mutant background, could be due to an indirect compensatory mechanism that enhances $A c n B$ synthesis during severe oxidative stress. Expression of the acnB-lacZ fusion was lowered to a limited extent during iron-depletion with bipyridyl (Fig. 5c). However, the failure of the multicopy $\mathrm{fur}^{+}$plasmid to reverse the small derepression observed in the fur mutant indicated that $a c n B$ is neither activated nor repressed by Fur.

Regulation of acn gene expression by ArcA and FNR. Studies with an acnA-lacZ fusion previously showed that the acnA gene is anaerobically repressed by ArcA (Gruer \& Guest, 1994; Gruer et al., 1997b). However, this repression was only partially reversed $(38 \%)$ by deleting the $\operatorname{arc} A$ gene, suggesting that some other mechanism of anaerobic repression might be operating. It was also concluded that any involvement of FNR must be indirect (or operational only in the absence of ArcA), because acnA expression was not enhanced in an $f n r$ mutant, but it was partially repressed under aerobic conditions by $\mathrm{fnr}^{+}$amplification in an $\operatorname{arcA} A^{+}$strain (Gruer \& Guest, 1994). When further investigated with an arcA fnr double mutant, it was found that the aerobic expression of the acn $A-l a c Z$ fusion is partially and equally repressed (to $42 \%$ of the parental level) by multiple copies of either regulatory gene, $\operatorname{arcA}^{+}$or $\mathrm{fnr}^{+}$(Fig. 6a). However, the severe anaerobic repression in the parental strain was almost fully $(92 \%)$ reversed in the double mutant but reimposed by multiple copies of each regulatory gene (Fig. 6a). This indicates that acnA expression is anaerobically repressible by ArcA and FNR, the effect of FNR being masked by ArcA in an $f n r$ 
(a) $a c n B(c r p)$

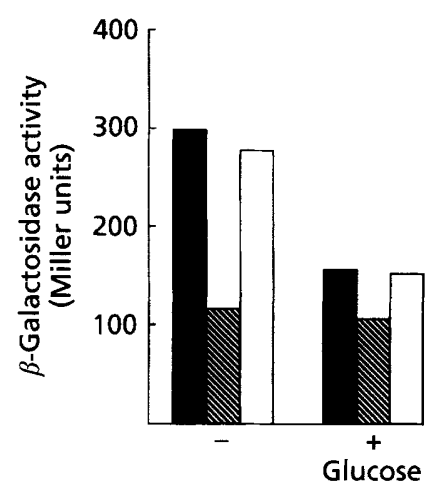

(b) $a C n B$ (soxRS)

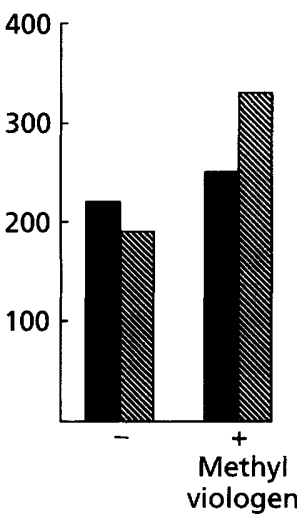

(c) $a c n B$ (fur)

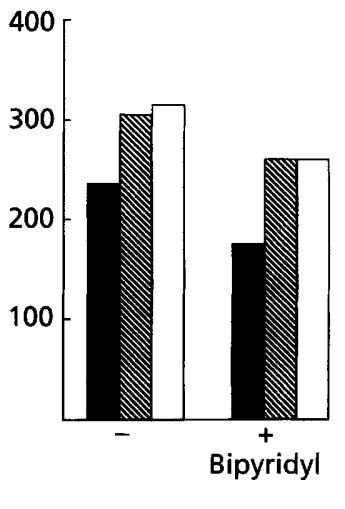

Fig. 5. Effects of CRP, SoxRS and Fur on acnB-lacZ expression. The $\beta$-galactosidase activities of aerobically grown lateexponential-phase L-broth cultures of $\lambda$ acnB-lacZ ( $\lambda \mathrm{G} 259)$ monolysogens having wild-type $(\mathbb{E})$, regulator mutant $(\mathbb{Q})$, and mutant plus multicopy regulator plasmid $(\square)$ backgrounds, were compared for the effects of: (a) $c r p$; glucose $(1 \%, w / v)$ was added to the growth medium where indicated; (b) SoxRS; redox-stress was imposed by adding methyl viologen $(0.2 \mathrm{mg}$ $\mathrm{ml}^{-1}$ ) $1 \mathrm{~h}$ before harvesting; and (c) fur; iron was depleted by adding bipyridyl $(200 \mu \mathrm{M})$ $1 \mathrm{~h}$ before harvesting where indicated. (a) acnA (arcA fnr)

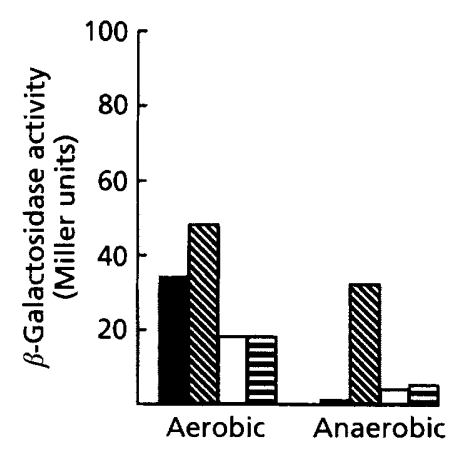

(b) $\operatorname{acn} B(\operatorname{arc} A)$

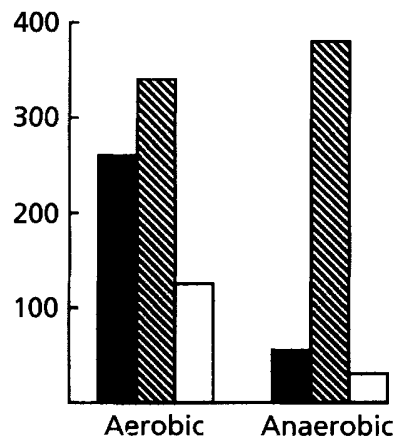

(c) $a c n B(f n r)$

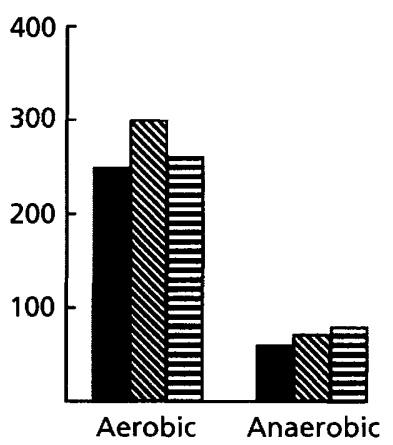

(d) $\operatorname{acnB}(\operatorname{arc} A$ fnr)

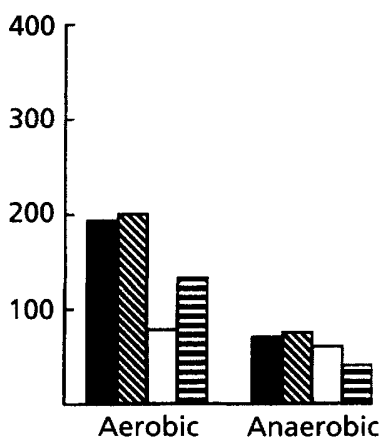

Fig. 6. Effects of ArcA and FNR on acnA-lacZ and acnB-lacZ expression. $\beta$-Galactosidase activities of stationary-phase cultures of $\lambda$ acnA-lac $Z$ ( $\lambda$ G244) monolysogens, and late-exponential-phase cultures of $\lambda$ acnB-lacZ ( $\lambda$ G259) monolysogens, were compared after aerobic and anaerobic growth in $L$ broth. The fusion prophages were established in wild-type ( $\square$ ), $\operatorname{arc} A$ and $f n r$ single or double mutant $(\mathbb{\nabla})$, and mutant containing multicopy $\operatorname{arc}^{+}$( $\square$ ) or $f n r^{+}$(目) plasmids, as backgrounds for studying the effects of: (a) arcA fnr double mutation on acnA-lacZ expression; (b) arcA on acnB-lacZ; (c) fnr on $a c n B-l a c Z$; and (d) arcA fnr double mutation on acnB-lacZ expression.

mutant but more apparent in an arcA mutant. As concluded previously by Gruer \& Guest (1994), the effect of FNR is not simply due to its known ability to activate $\operatorname{arcA}$ transcription (Compan \& Touati, 1994).

The responses obtained with the acnB-lac $Z$ fusion indicated that ArcA functions as an anaerobic repressor of $a c n B$ transcription and that the $a c n B$ gene is not regulated by FNR. The aerobic expression was seemingly poised in the working range of ArcA because the activity was enhanced $1 \cdot 25$-fold in the $\operatorname{arcA}$ deletion strain but repressed 2-fold in the multicopy arcA host (Fig. 6b). The parental activity was anaerobically repressed $4 \cdot 3$-fold and further repressed by multicopy $\operatorname{arcA} A^{+}$, but this repression was abolished in the $\operatorname{arcA}$ deletion strain (Fig. 6b). In contrast, the effects of inactivating or amplifying the $\mathrm{fnr}^{+}$gene were insignificant under aerobic and anaerobic conditions (Fig. 6c). However, this simple picture of ArcA-mediated anaerobic repression was complicated by the behaviour of the $a c n B-l a c Z$ fusion in the $\operatorname{arc} A$ fnr double mutant (Fig. 6d). First, there was no significant anaerobic derepression of $a c n B-l a c Z$ activity in the double mutant, suggesting that in the absence of both regulators, some other repressor might invade the $a c n B$ promoter. Second, multiple copies of the $\mathrm{fnr}^{+}$gene lowered aerobic and anaerobic expression to $68 \%$ and $58 \%$ of the respective parental levels, suggesting that in the absence of ArcA, amplified FNR can interact negatively at the $a c n B$ promoter.

Regulation of the acn and fum gene expression by $\sigma^{38}$. The role of $\sigma^{38}$ in aconitase gene expression was investigated because acnA is not maximally expressed until stationary phase (Gruer et al., 1997). Accordingly, it was found that acnA-lacZ expression is lowered fivefold by disrupting the $r p o S$ gene and restored by a multicopy $r p o S^{+}$ plasmid (Fig. 7a), indicating that the $\operatorname{acn} A$ gene is subject to $\sigma^{38}$-mediated transcription initiation. In contrast, expression of the $a c n B-l a c Z$ fusion was enhanced $2 \cdot 4$-fold in the rpoS mutant and restored to the parental level by the multicopy rpoS $S^{+}$plasmid (Fig. 7b). The apparent negative control of $a c n B$ by $\sigma^{38}$ will be discussed below. 
(a) $\operatorname{acnA}(r p o s)$

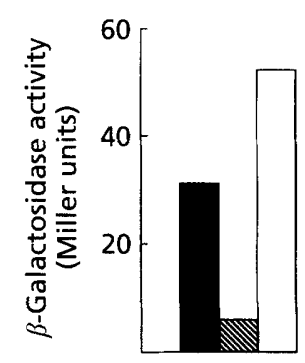

(b) $a c n B(r p o S)$

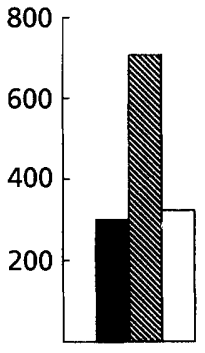

(c) fumA (rpos)

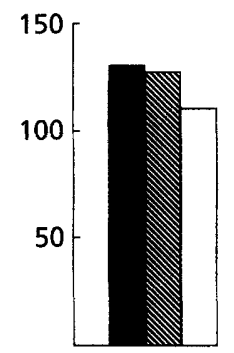

(d) fumC (rpos)

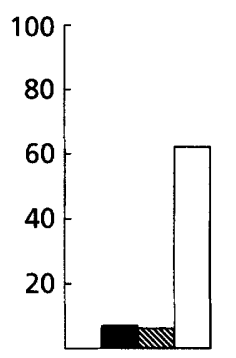

Fig. 7. Effects of $\sigma^{38}$ on the expression of single-copy acnA-, acnB-, fumA- and fumC-lacZ fusions. The $\beta$-galactosidase activities were assayed after aerobic growth in $L$ broth. Monolysogenic derivatives of a parental strain ( $(\mathbf{D})$, an rpoS mutant $(\mathbb{S})$ and an rpos mutant transformed with a multicopy rpos $^{+}$plasmid $(\square)$, contained the following fusion prophages: (a) $\lambda a c n A-l a c Z$;

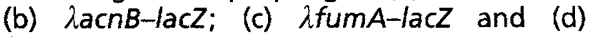
$\lambda$ fumC-lacZ. (a) acnA (fis)

(b) $a c n B$ (fis)

(c) $\operatorname{acn} A($ fruR)

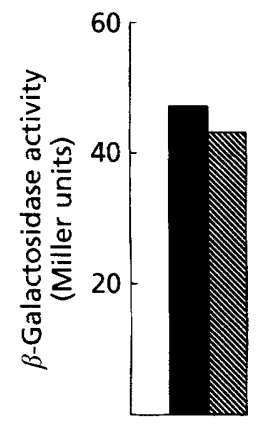

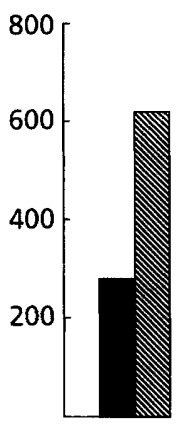

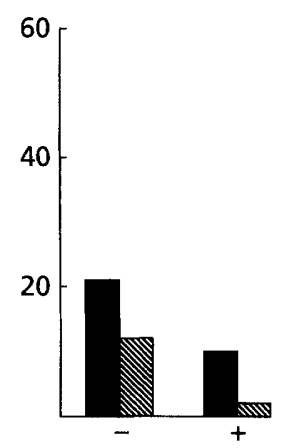

Glucose (d) $a c n B$ (fruR)

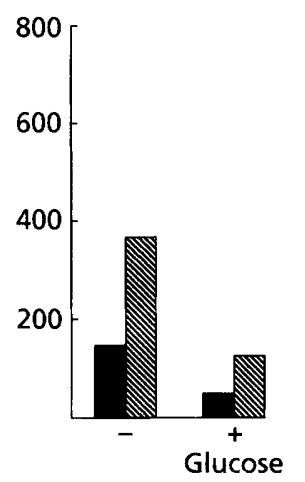

Fig. 8. Effects of Fis and FruR on the expression of single-copy acnA- and acnB-lac $Z$ fusions. The $\beta$-galactosidase activities were assayed after aerobic growth in $L$ broth (fis) or in $L$ broth with $(+)$ or without $(-)$ glucose (fruR). Monolysogenic derivatives of parental ( $\square$ ) and mutant strains $(\mathbb{\nabla})$ were compared for the effects of: (a) fis on acnA-lacZ; (b) fis on acnB-lacZ; (c) fruR on acnA-lacZ; and (d) fruR on $a c n B-l a c Z$ expression.
The present studies were extended to include the fum $A$ and $f u m B$ genes which encode analogous [4Fe-4S]containing aerobic and anaerobic fumarases (FumA and FumB, respectively), and the fum $C$ gene, which encodes an oxygen-stable enzyme (FumC) that closely resembles the mitochondrial fumarases (Woods et al., 1986; Bell et al., 1989). The patterns of activity observed with the fum-lac $Z$ fusions indicated that fumA expression is $\sigma^{38}$. independent (Fig. 7c). The very low aerobic expression of the fumB-lacZ fusion was likewise unaffected by $\sigma^{38}$ (data not shown). However, despite its apparent $\sigma^{38}$. independence, expression of the fum $\mathrm{C}$ fusion was activated ninefold by amplifying $\sigma^{38}$ (Fig. 7d).

Regulation of acn gene expression by Fis. The effect of Fis on acn gene expression was examined because Fisregulated genes are, like $a c n B$, strongly expressed in mid-exponential phase, and also because Fis has been shown to repress the succinate dehydrogenase $(s d h)$ and $\mathrm{NADH}$ dehydrogenase II $(n d h)$ genes (Finkel \& Johnson, 1992; Xu \& Johnson, 1995; Green et al., 1996). Deleting the fis gene had no effect on acnA-lacZ expression (Fig. 8a) but it was accompanied by a twofold increase in $a c n B-l a c Z$ expression (Fig. 8b), indicating that $a \subset n B$ is repressed by Fis.

Regulation of acn gene expression by FruR. FruR (also known as Cra) regulates the expression of many genes involved in carbon metabolism. Thus, it activates the isocitrate lyase, malate synthase and isocitrate dehydrogenase genes, and represses those encoding glycolytic enzymes (Ramseier et al., 1993; Saier \& Ramseier, 1996). Studies with the acn-lacZ fusions indicated that
acnA is subject to Fru-mediated activation (Fig. 8c), whereas the $a c n B$ gene is repressed by FruR (Fig. 8d). Furthermore, these effects were still observed in the presence of glucose, which is presumed to abolish CRPmediated activation. Indeed, the combined effect of eliminating FruR- and CRP-mediated activation was a 10 -fold lowering of $a c n A$ expression (Fig. 8c). Conversely, $a c n B$ expression was enhanced eightfold by abolishing FruR- and glucose-mediated repression (Fig. 8d).

\section{DISCUSSION}

The factors controlling the expression of the acnA and $a c n B$ genes of $E$. coli are summarized in Fig. 9. Both genes are subject to ArcA-mediated anaerobic repression and CRP-mediated catabolite repression, but they differ insofar as acnA gene transcription is activated by Fur, SoxRS and FruR, can be initiated by $\sigma^{38}$, and is repressed by FNR, whereas the $a c n B$ gene is repressed by Fis and FruR but unaffected by the other regulators. The acn $A$ gene is only weakly expressed compared to the $a c n B$ gene, even after redox-stress induction. This is reflected in the relatively small effect of acnA mutation on aconitase specific activity compared with that inflicted by $a c n B$ mutation (Gruer et al., 1997b). It is also consistent with the observation that using Northern blotting, acnA transcripts $(2 \cdot 8 \mathrm{~kb})$ could only be detected in redox-stressed cultures. These transcripts were correlated with one of the two acnA promoters ( $\left.P 2_{\text {acnA }}\right)$ which is responsible for the basal level of acnA transcription that occurs throughout the growth cycle as well as that induced by redox-stress (Table 2; Figs 4a, 9). 

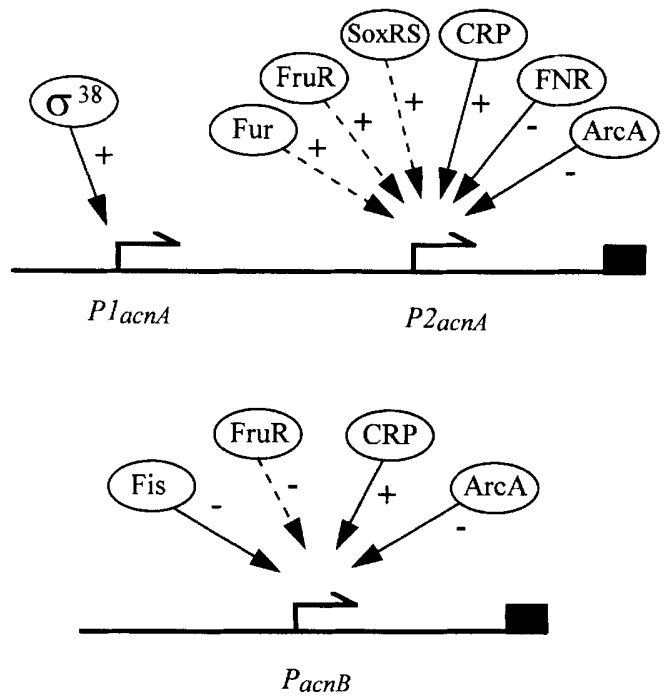

Fig. 9. Regulation of the aconitase genes of $E$. coli. The effects of global regulators on the activities of specific promoters, (a) $\operatorname{acn} A$ and (b) $a c n B$, are indicated. The broken arrows denote that the involvement of the regulator may be indirect.

Although no transcript initiating at $P 1_{\mathrm{acnA}}$ could be detected by Northern blotting, this upstream promoter is apparently responsible for the increase in acnA expression that occurs as cultures enter stationary phase (Table 2). In contrast, $a c n B$ gene expression was solely dependent on transcription from a single promoter, $P_{\text {acnB }}$ (Figs $4 b, 9$ ).

\section{Regulation of acnA gene transcription}

The upstream acnA promoter $\left(P 1_{\mathrm{acnA}}\right)$ resembles a socalled gearbox promoter, which often controls genes whose transcription is inversely proportional to growth rate and are positively regulated by $\sigma^{38}$ (Vicente et al., 1991). Furthermore, $\sigma^{38}$ is an alternative sigma factor used for the activation of genes concerned with general stress conditions as well as stationary-phase survival (Hengge-Aronis, 1993, 1996). The $\sigma^{38}$-dependent activation of acnA transcription from $P 1_{\mathrm{acnA}}$ may thus form part of a more general stress-induced response.

The second promoter $\left(P 2_{\mathrm{acnA}}\right)$ more typically resembles a factor-dependent $\sigma^{70}$ promoter, by virtue of its poor -35 region (Fig. 4a). This promoter is the site of SoxRSmediated activation, but the absence of a convincing SoxS binding site (Li \& Demple, 1996) upstream of $P 2_{\text {acn A }}$, suggests that the mode of activation might be indirect. There is a potential CRP site centred at -40.5 , resembling a CRP (class II) activated promoter (Busby \& Kolb, 1996; Fig. 4a), which could account for the observed CRP-dependence of $\operatorname{arcA}$ expression. This site is overlapped by potential ArcA and FNR binding sites (centred at -37.5 and -40.5 , respectively). Attention has previously been drawn to this ArcA site by Lynch \& Lin (1996) even though there are a further 10 potential
ArcA sites matching the consensus at $\geqslant 7$ out of 10 positions, in the untranscribed intergenic region. It is not known which are responsible for the well-established ArcA-mediated anaerobic repression of acnA (Gruer \& Guest, 1994). The repressing role of FNR that operates in the absence of ArcA was here confirmed with an $\operatorname{arc} A$ fnr double mutant. It might be mediated by the overlapping arrangement of the two binding sites, if ArcA has the higher affinity for this region and thus normally displaces FNR (Fig. 4a). However, the situation may be more complex, because FNR centred at -40.5 might be expected to activate rather than repress, and because FNR-mediated repression often involves FNR interactions at two or more sites (Guest et al., 1996). It is therefore possible that other potential FNR sites centred at -76.5 and -247.5 (Fig. $4 a$ ) may be associated with the observed repression. Whether FNR performs a modulating or other role in the regulation of $\operatorname{acn} A$ expression in an $\operatorname{arc} A^{+}$strain background has yet to be determined.

The presence of potential Fur sites (centred at -321 , $-318,-315$ and -229 relative to the $P 2_{\text {aena }}$ transcriptional start site) seemed to be consistent with the established Fur- and iron-dependence of $\operatorname{acn} A$ gene expression (Gruer \& Guest, 1994). However, despite matching the Fur site consensus at 12-14 out of 19 positions, recent searches using a scoring matrix assembled from 16 E. coli Fur sites revealed no significant matches. It is therefore concluded that the regulation of $\operatorname{acn} A$ by Fur is indirect. FruR (Cra) is a transcriptional regulator which, together with CRP, controls the expression of genes involved in $\mathrm{C}$ metabolism, particularly with respect to the direction of metabolic flow, glycolysis being repressed whereas gluconeogenesis is activated (Ramseier et al., 1993; Saier \& Ramseier, 1996). The acnA gene, like genes encoding some other citric acid and glyoxylate cycle enzymes, is activated by FruR. However, it is significant that $a c n B$ is repressed by FruR (see below): no potential FruR binding sites were detected in either promoter region.

\section{Regulation of $a c n B$ gene transcription}

Apart from being activated by CRP and repressed by ArcA, the $a c n B$ gene exhibited a very different pattern of regulation to that of $a c n A$. The promoter region contains only one potential CRP binding site, centred at a position $(-37.5)$ that is normally associated with repression rather than activation (Fig. 4b; Busby $\&$ Kolb, 1996). In contrast, there are many putative ArcA sites, which match the binding site consensus at 7-8 out of 10 positions (Lynch \& Lin, 1996) and could thus be associated with the ArcA-mediated anaerobic repression of $a c n B$. Seven such sites are shown in Fig. 4(b) and there are three more located further upstream. The observed decline in $a c n B$ expression and $A c n B$ activity observed in stationary phase (Gruer et al., 1997b) is interesting because it could be related to the ArcA-mediated repression of citric acid cycle enzymes during carbon starvation, as part of a general survival strategy aimed at 
conserving endogenous reserves and limiting the production of oxygen radicals by continued aerobic respiratory activity (Nyström et al., 1996).

The observed derepression of $a c n B$ in the rpoS mutant and reversal by the $r p o S^{+}$plasmid (Fig. 7b) suggests that the $a c n B$ gene is repressed by $\sigma^{38}$. Possible explanations are that: (i) free $\sigma^{38}$ could bind directly to promoter DNA, as has been shown for the $\sigma^{54}$ protein (Buck \& Cannon, 1992); (ii) RNA polymerase core enzyme, no longer bound to $\sigma^{38}$ in the rpoS mutant, may become available for use with $\sigma^{70}$ and thus enhance transcription at $P_{\mathrm{acnB}}$ and other $\sigma^{70}$ promoters; or (iii) $\sigma^{38}$ polymerase may function indirectly by activating the synthesis of an $a c n B$ repressor. The reason why FruR represses $a c n B$ but activates acn $A$ is unclear. It may be related to the preferential use of one of the two enzymes in the citric acid and glyoxylate cycles or in stress responses. Fis (the factor for inversion stimulation) was originally identified by virtue of its role in site-specific DNA inversion, but is now increasingly associated with the activation and repression of gene expression, presumably via its ability to induce DNA-bending (Xu \& Johnson, 1995). The $a c n B$ promoter region contains three potential Fis binding sites (Fig. 4b). One of the sites overlaps the -35 hexamer, but occluding RNA polymerase is not the only mechanism that has been proposed for Fis-mediated repression.

The existence of two aerobic but differentially regulated aconitases parallels that of the fumarases, where there are major (FumA) and minor (FumC) aerobic enzymes, encoded by adjacent genes (fumA-fumC), and an anaerobic enzyme (FumB). It is now apparent from studies with the corresponding lac $Z$ fusions that the fumA promoter resembles $P_{\mathrm{acnB}}$ in being anaerobically repressed by $\mathrm{ArcA}$, whereas the fum $\mathrm{C}$ promoter resembles $P 2_{\mathrm{acnA}}$ in its dual regulation by ArcA and FNR, although there is evidence that fum $C$ transcription is partially facilitated by readthrough from the upstream fumA gene promoter (Woods \& Guest, 1987; Guest \& Russell, 1992; Gruer \& Guest, 1994; Park \& Gunsalus, 1995). The relationship is further strengthened by showing that the genes ( $f u m C$ and $\operatorname{acn} A$ ) encoding the minor aerobic enzymes are alone activated by SoxRS and $\sigma^{38}$. However, it breaks down with respect to Fur, which activates the minor aconitase gene $(\operatorname{acn} A)$ but not the minor fumarase gene (fumC) except by readthrough transcription from its primary activation target, the gene encoding the major fumarase (fumA). Nevertheless, it is clear that for aconitase and fumarase, there are major aerobic enzymes (AcnB and FumA) whose transcriptional regulation is typical of citric acid cycle enzymes, whereas the minor enzymes (AcnA and FumC) are adapted for stress-related responses (activation by SoxRS and transcription by $\sigma^{38}$ ). Current work is aimed at characterizing the putative third aconitase $(A c n C$ ), the minor residual aconitase activity of an $a c n A a c n B$ double mutant, and ascertaining whether AcnA (or $A c n B)$ perform iron-dependent regulatory functions analogous to mammalian IRP1.

\section{ACKNOWLEDGEMENTS}

We are very grateful to Drs J. Green, S. C. Andrews and M. A. Quail and for advice and helpful discussions. This work was supported by the Wellcome Trust.

\section{REFERENCES}

Aiba, H., Adhya, S. \& de Crombrugghe, B. (1981). Evidence for two functional gal promoters in intact Escherichia coli cells. J Biol Chem 256, 11905-11910.

Bartolomé, B., Jubete, Y., Martinez, E. \& de la Cruz, F. (1991). Construction and properties of a family of pACYC184-derived cloning vectors compatible with $\mathrm{pBR} 322$ and its derivatives. Gene $102,75-78$.

Beinert, H., Kennedy, M. C. \& Stout, C. D. (1996). Aconitase as iron-sulfur protein, enzyme, and iron regulatory protein. Chem Rev 96, 2335-2373.

Bell, P. J., Andrews, S. C., Sivak, M. N. \& Guest, J. R. (1989). Nucleotide sequence of the FNR-regulated fumarase gene ( $(\mathrm{umB})$ of Escherichia coli K12. J Bacteriol 171, 3494-3503.

Bradbury, A. J., Gruer, M. J., Rudd, K. E. \& Guest, J. R. (1996). The second aconitase (AcnB) of Escherichia coli. Microbiology 142, 389-400.

Busby, S. \& Kolb, A. (1996). The CAP modulon. In Regulation of Gene Expression in Escherichia coli, pp. 255-279. Edited by E. C. C. Lin \& S. A. Lynch. Austin: R. G. Landes.

Buck, M. \& Canon, W. (1992). Specific binding of the transcription factor sigma-54 to promoter DNA. Nature 358, 422-424.

Chamberlin, M. J. \& Hsu, L. M. (1996). RNA chain initiation and promoter escape by RNA polymerase. In: Regulation of Gene Expression in Escherichia coli, pp. 7-25. Edited by E. C. C. Lin \& S. A. Lynch. Austin: R. G. Landes.

Compan, I. \& Touati, D. (1994). Anaerobic activation of $\operatorname{arcA}$ transcription in Escherichia coli: roles for Fnr and ArcA. Mol Microbiol 11, 955-964.

Finkel, S. E. \& Johnson, R. C. (1992). The Fis protein: it's not just for DNA inversion anymore. Mol Microbiol 6, 3257-3265.

Frishman, D. \& Hentze, M. W. (1996). Conservation of aconitase residues revealed by multiple sequence analysis. Implications for structure/function relationships. Eur J Biochem 239, 197-200.

Fujita, N., Mori, H., Yur, T. \& Ishihama, A. (1994). Systematic sequencing of the Escherichia coli genome-analysis of the 2.4-4.1 min (110,917-193,643 bp) region. Nucleic Acids Res 22, 1637-1639.

Gerischer, U. \& Durre, P. (1992). mRNA analysis of the adc gene region of Clostridium acetobutylicum during the shift to solventogenesis. J Bacteriol 174, 426-433.

Green, J., Anjum, M. F. \& Guest, J. R. (1996). The $n d h$-binding protein (Nbp) regulates the $n d b$ gene of Escherichia coli in response to growth phase and is identical to Fis. Mol Microbiol 20, $1043-1055$.

Greenberg, J. T., Monach, P., Chou, J. H., Josephy, D. \& Demple, B. (1990). Positive control of a global antioxidant defense regulon activated by superoxide generating agents in Escherichia coli. Proc Natl Acad Sci USA 87, 6181-6185.

Gruer, M. J. \& Guest, J. R. (1994). Two genetically distinct and differentially regulated aconitases (AcnA and AcnB) in Escherichia coli. Microbiology 140, 2531-2541.

Gruer, M. J., Artymiuk, P. J. \& Guest, J. R. (1997a). The aconitase family: three structural variations on a common theme. Trends Biochem Sci 22, 1-4. 
Gruer, M. J., Bradbury, A. J. \& Guest, J. R. (1997b). Construction and properties of aconitase mutants of Escherichia coli. Microbiology 143, 1837-1846.

Guest, J. R. \& Russell, G. C. (1992). Complexes and complexities of the citric acid cycle in Escherichia coli. Curr Top Cell Regul 33, 231-247.

Guest, J. R., Green, J., Irvine, A. S. \& Spiro, S. (1996). The FNR modulon and FNR regulates gene expression. In: Regulation of Gene Expression in Escherichia coli, pp. 317-342. Edited by E. C. C. Lin \& S. A. Lynch. Austin: R. G. Landes.

Hengge-Aronis, R. (1993). Survival of hunger and stress: the role of $r p o S$ in early stationary phase gene regulation in Escherichia coli. Cell 72, 165-168.

Hengge-Aronis, R. (1996). Back to log phase: $\sigma^{\mathrm{s}}$ as a global regulator in the osmotic control of gene expression in Escherichia coli. Mol Microbiol 21, 887-893.

Hentze, M. W. \& Kuhn, L. C. (1996). Molecular control of vertebrate iron metabolism - messenger RNA based regulatory circuits operated by iron, nitric oxide and oxidative stress. Proc Natl Acad Sci USA 93, 8175-8182.

Klausner, R. D. \& Rouault, T. A. (1993). A double life - cytosolic aconitase as a regulatory RNA-binding protein. Mol Biol Cell 4, $1-5$.

Kumar, A., Malloch, R. A., Fujita, N., Smillie, D. A., Ishihama, A. \& Hayward, R. S. (1993). The -35 recognition region of Escherichia coli $\sigma^{70}$ is inessential for initiation of transcription at an 'extended - 10' promoter. J Mol Biol 232, 406-418.

Li, Z. \& Demple, B. (1996). Sequence specificity for DNA binding by Escherichia coli SoxS and Rob proteins. Mol Microbiol 20, 937-945.

Lynch, A. S. \& Lin, E. C. C. (1996). Transcriptional control mediated by the ArcA two component response regulator protein of Escherichia coli: characterization of DNA binding at target promoters. J Bacteriol 178, 6238-6249.

Miller, J. H. (1972). Experiments in Molecular Genetics. Cold Spring Harbor, NY: Cold Spring Harbor Laboratory.

Mulvey, M. R., Sorby, P. A., Triggs-Raine, B. L. \& Loewen, P. C. (1988). Cloning and physical characterization of katE and katF required for catalase HPII expression in Escherichia coli. Gene 73, $337-345$.

Nyström, T., Larsson, C. \& Gustafsson, L. (1996). Bacterial defence against aging: role of the Escherichia coli ArcA regulator in gene expression, readjusted energy flux and survival during stasis. EMBO J 15, 3219-3228.

Park, S. J. \& Gunsalus, R. P. (1995). Oxygen, iron, carbon, and superoxide control of the fumarase fumA and fumC genes of Escherichia coli: role of the $\operatorname{arcA}$, fnr, and soxR gene products. $J$ Bacteriol 177, 6255-6262.
Prodromou, C., Haynes, M. J. \& Guest, J. R. (1991). The aconitase of Escherichia coli: purification of the enzyme and molecular cloning and map location of the gene (acn). J Gen Microbiol 137, 2505-2515.

Prodromou, C., Artymiuk, P. J. \& Guest, J. R. (1992). The aconitase of Escherichia coli. Eur J Biochem 204, 599-609.

Ramseier, T. M., Negre, N., Cortay, J. C., Scarabel, M., Cozzone, A. J. \& Saier, M. H. (1993). In vitro binding of the pleiotropic transcriptional regulatory protein, FruR, to the $f r u, p p s$, ace, $p t s$, and icd operons of Escherichia coli and Salmonella typhimurium. $J$ Mol Biol 234, 28-44.

Robbins, A. H. \& Stout, C. D. (1989). The structure of aconitase. Proteins 5, 289-312.

Saier, M. H. \& Ramseier, T. M. (1996). The catabolite repressor/ activator (Cra) protein of enteric bacteria. J Bacteriol 178, 3411-3417.

Sambrook, J., Fritsch, E. F. \& Maniatis, T. (1989). Molecular Cloning: a Laboratory Manual, 2nd edn. Cold Spring Harbor, NY: Cold Spring Harbor Laboratory.

Sawers, G. \& Suppmann, B. (1992). Anaerobic induction of pyruvate formate-lyase gene expression is mediated by the ArcA and FNR proteins. J Bacteriol 174, 3474-3478.

Silhavy, T. J., Barman, M. L. \& Enquist, L. W. (1984). Experiments with Gene Fusions. Cold Spring Harbor, NY: Cold Spring Harbor Laboratory.

Spiro, S., Roberts, R. E. \& Guest, J. R. (1989). FNR-dependent repression of the $n d h$ gene of Escherichia coli and metal ion requirement for FNR-regulated gene expression. Mol Microbiol 3, 601-608.

Vicente, M., Kushner, S. R., Garrido, T. \& Aldea, M. (1991). The role of the gearbox in the transcription of essential genes. $\mathrm{Mol}$ Microbiol 5, 2085-2091.

Woods, S. A., Miles, J. S., Roberts, R. E. \& Guest, J. R. (1986). Structural and functional relationships between fumarase and aspartase: nucleotide sequences of the fumarase (fumC) and aspartase (aspA) genes of Escherichia coli K12. Biochem J 237, $547-557$.

Woods, S. A. \& Guest, J. R. (1987). Differential roles of the Escherichia coli fumarases and $f n r$-dependent expression of fumarase B and aspartase. FEMS Microbiol Lett 48, 219-224.

$\mathrm{Xu}$, J. \& Johnson, R. C. (1995). Identification of genes negatively regulated by Fis: Fis and RpoS co-modulate growth-phasedependent gene expression in Escherichia coli. J Bacteriol 177, 938-947.

Received 20 June1997; revised 7 August 1997; accepted 12 August 1997. 